\title{
LRH-1 High Expression in the Ovarian Granulosa Cells of PCOS Patients
}

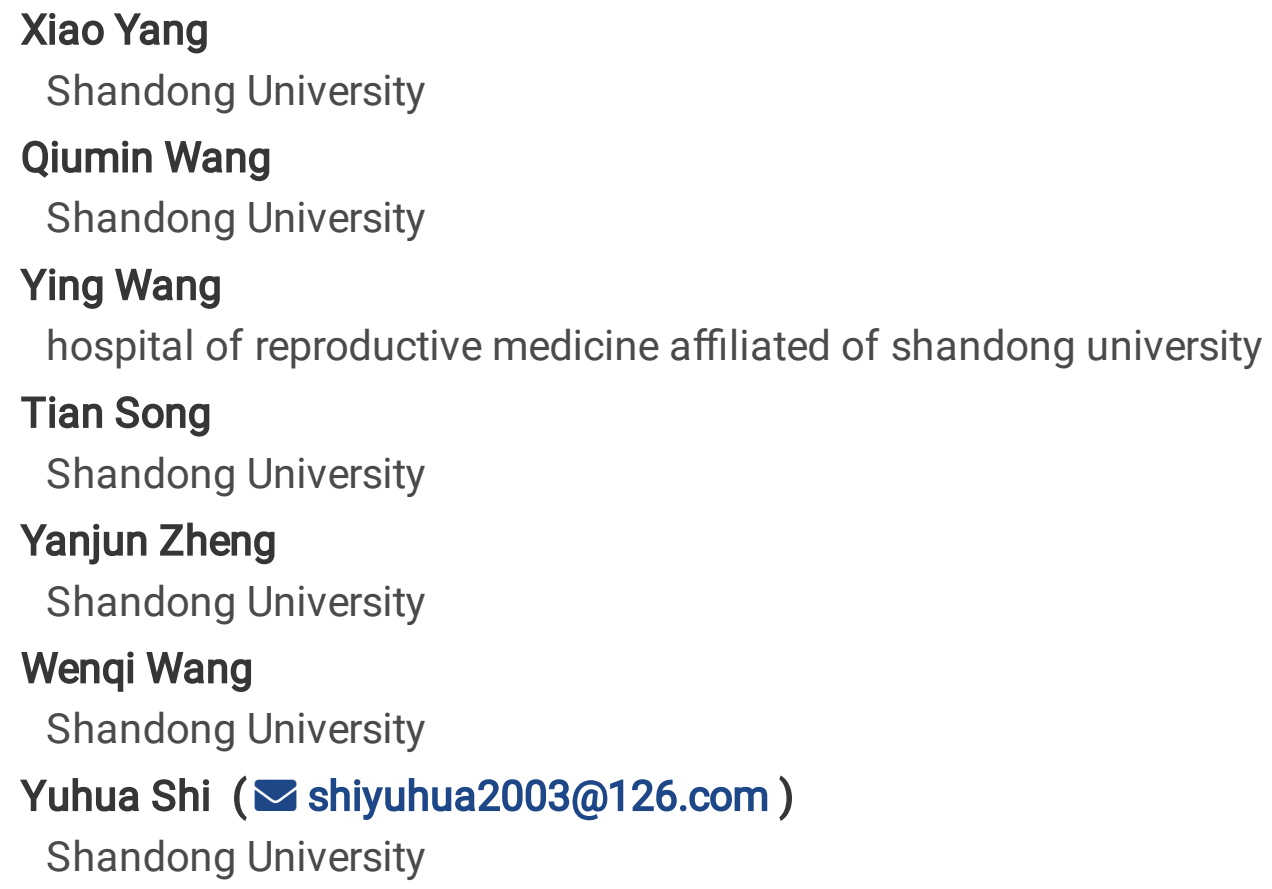

Xiao Yang

Shandong University

Qiumin Wang

Shandong University

Ying Wang

hospital of reproductive medicine affiliated of shandong university

Tian Song

Shandong University

\section{Yanjun Zheng}

Shandong University

Wenqi Wang

Shandong University

Yuhua Shi ( $\sim$ shiyuhua2003@126.com)

Shandong University

\section{Research Article}

Keywords: polycystic ovary syndrome, liver receptor homolog 1, anovulation, granulosa cells

Posted Date: March 30th, 2021

DOI: https://doi.org/10.21203/rs.3.rs-342893/v1

License: (c) (i) This work is licensed under a Creative Commons Attribution 4.0 International License.

Read Full License 


\title{
LRH-1 high expression in the ovarian granulosa cells of PCOS patients
}

Xiao Yang*1,2,3,4,5, Qiumin Wang ${ }^{* 1,2,3,4,5}$, Ying Wang*1,2,3,4,5 ,Tian Song 1,2,3,4,5, Yanjun Zheng ${ }^{1,2,3,4,5}$, Wenqi Wang ${ }^{1,2,3,4,5}$,Yuhua Shi ${ }^{1,2,3,4,5}$

*Xiao Yang, Qiumin Wang, Ying Wang contributed equally to this article.

Corresponding Author: Shi Yuhua 1,2,3,4,5, shiyuhua2003@126.com

1.Center for Reproductive Medicine, Cheeloo College of Medicine, Shandong University, Jinan,Shandong, 250012, China

2.Key laboratory of Reproductive Endocrinology of Ministry of Education, Shandong University, Jinan, Shandong, 250012, China

3.Shandong Key Laboratory of Reproductive Medicine, Jinan, Shandong, 250012, China

4. Shandong Provincial Clinical Research Center for Reproductive Health, Jinan, Shandong, 250012 , China

5.National Research Center for Assisted Reproductive Technology and Reproductive Genetics, Shandong University, Jinan, Shandong, 250012, China

\begin{abstract}
Purpose : Polycystic ovary syndrome (PCOS) is considered as one of the most common endocrine disorder with heterogeneity. There are also reports that liver receptor homolog 1 [LRH-1 or nuclear receptor subfamily 5 group A member 2 (NR5A2)] plays an important role in the reproductive system. But up to now, there are no reports related to the link with PCOS and LRH-1. In this study, we aimed to detect the LRH-1 expression in the ovarian granulosa cell of PCOS patients and explore the potential relationship between LRH-1 and PCOS.

Methods : 146 follicular fluid sample were collected in this study, including 72 from PCOS patients and 74 from control patients who underwent intracytoplasmic sperm injection (ICSI) or in vitro fertilization-embryo transfer (IVF-ET). The ovarian granulosa cells were extracted from the patient's follicular fluid by magnetic-activated cell sorting (MACS) method, and the real-time quantitative PCR (qRT-PCR) was used to measure the expression of LRH-1 in ovarian granulosa cells. Then we analyzed the correlation between the expression level of LRH-1 and the clinical characteristics of patient by using Pearson Correlation analysis.

Results: The expression of LRH-1 was significantly higher in PCOS patients ovarian granulosa cells than that in the control patients $[\operatorname{vs}(1.38 \pm 0.47) \operatorname{vs}(1.03 \pm 0.32), \mathrm{t}=5.327, \mathrm{p}<0.0001]$, and it was positively correlated with antral follicles counting (AFC) $(\mathrm{r}=0.3607, \mathrm{p}<0.0001)$ and the serum $\mathrm{AMH}(\mathrm{r}=0.2662$, $\mathrm{p}=0.0012) \backslash \mathrm{LH}(\mathrm{r}=0.2518, \mathrm{p}=0.0022) \backslash \mathrm{T}(\mathrm{r}=0.2516, \mathrm{p}=0.0022)$ in all patients. No statistical significance between LRH-1 and BMI, FSH, HOMA-IR, DHE-S, progesterone.
\end{abstract}


Conclusions : Compared with the control group, we found that LRH-1 was highly expressed in the ovarian granulosa cells of PCOS patients. Our study has revealed the relationship between the LRH-1 expression and PCOS, which suggested that LRH-1 may play an important role in ovulation disorders. While this finding provided new ideas for the study of the pathogenesis, it also provided a theoretical basis for the clinical diagnosis and treatment for PCOS.

\section{Keywords}

polycystic ovary syndrome; liver receptor homolog 1; anovulation; granulosa cells

\section{Declarations}

\section{Funding}

This study was supported by National Key R\&D Program of China (2018YFC1003202, 2017YFC1001004) and Taishan scholar project special funds (No. ts201712103).

\section{Conflicts of interest}

The authors declare that they have no competing interests.

\section{Ethics approval}

The institutional review board of the Center for Reproductive Medicine, Shandong University approved this study

\section{Consent for publication}

All authors gave their final approval to the publication of the manuscript.

\section{Availability of data and material}

The data and materials are available from the corresponding author on reasonable requests.

\section{Authors' contributions}

Xiao Yang, Qiumin Wang, Ying Wang contributed equally to this article. Xiao Yang and Yuhua Shi conceived, designed this study; Ying Wang, Tian Song and Wenqi Wang collected follicular fluid samples and clinical data; Xiao Yang, Qiumin Wang and Yanjun Zheng contributed to statistical analysis; Xiao Yang drafted the manuscript; Qiumin Wang, Ying Wang and Yuhua Shi participated in the discussion and critically revised the article.

\section{Introduction}


PCOS is a common type of endocrine and metabolic disorders, with a prevalence of $6 \% \sim 20 \%$ in adolescent and women of childbearing age, which is the main cause of anovulatory infertility[1]. The clinical manifestations of patients with PCOS have a certain heterogeneity. The main clinical features include high androgen, ovulation disorders and ultrasound ovarian polycystic morphology. In the long term, compared with normal person, PCOS patients are more likely to occur the metabolic disorders, including the disorder of glucose metabolism and lipid metabolism, increasing the risk of developing type 2 diabetes(T2DM) and cardiovascular disease(CHD) [2] [3]. More and more evidences have suggested that PCOS a disease affected by genetic and environmental factors, but the specific pathogenesis of PCOS is still not clear presently.

LRH-1 is an orphan member of the nuclear receptor family of regulatory transcription factors[4], which is located on human and mouse chromosome 1[5]. In mammals, LRH-1 was reported highly expresses in the tissues with secretory function, such as the liver, pancreas, and intestine[6-8], playing an important role in the regulation of cholesterol metabolism and bile acid homeostasis. Meanwhile, the expression of LRH-1 was also found in the ovaries, endometrium and placenta, suggesting that LRH-1 may play an essential role in the reproductive system, such as regulating ovarian granulosa cells proliferation, stimulating the progesterone biosynthetic pathway during the differentiation of ovarian granulosa cells and affecting the development of the placenta[9-11].

Plenty of researchers have found that there is a correlation between the expression level of LRH-1 with tumorigenesis, such as ovarian cancer, non-small cell lung cancer and colon cancer [12-14]. At the same time, in the research of reproduction, it has been found that Unexplained Recurrent Spontaneous Abortion (URSA) was related to the decreased expression level of LRH-1 in villous tissues[15], and disruption of LRH-1 gene resulted in early embryonic death[16]. However, to date, there have been no study related to the relationship between the expression of LRH-1 and PCOS.

Therefore, in the present study, we focused on the relative expression level of LRH-1 in ovarian granulosa cells from patients with PCOS. Then we analyzed its correlation with clinical data, aiming to better understand the pathogenesis, as well as providing novel insight for clinical diagnosis and treatment of PCOS.

\section{Materials and methods}

\section{Clinical information of patients}

In this study, the clinical diagnosis of PCOS is based on the Rotterdam criteria which require to consisted with at least two of the following three conditions: clinical and / or biochemical evidence of hyperandrogenism (HA), oligo- and / or anovulation (OA), and polycystic ovarian morphology (PCOM) after excluding other etiologies such as Cushing's syndrome, congenital adrenal hyperplasia and androgen-secreting tumors[17]. We collected 72 follicular fluid sample from PCOS patients who underwent ICSI or IVF-ET in the Reproductive Hospital of Shandong University from December 2018 to September 2019. At the same time, 74 follicular fluid sample from patients with normal ovarian function undergoing IVF-ET/ICSI treatment due to tube infertility or male factors were collected as the control group. In order to eliminate the influence of obesity, the body mass index (BMI) of all patients was ranged from 18 to $30 \mathrm{~kg} / \mathrm{m}^{2}$. All participants were younger than 40 years old, excluding the history of ovarian surgery, reproductive system abnormalities, thyroid disease and chromosomal diseases that may affect pregnancy outcomes. Ovulation-stimulation regimen were either standard long agonist protocol or antagonist protocol under the guidance of experienced clinicians. The study was approved by the Ethics Committee of the Affiliated Reproductive Hospital of Shandong University. 


\section{Measurements of clinical indicators}

The clinical date contained at following: Age and BMI, the fasting insulin (FINS) and fasting plasma glucose (FPG) in venous blood in the state of $12 \mathrm{~h}$ overnight fasting, the levels of anti-Mullerian hormone (AMH), testosterone(T), follicle stimulating hormone (FSH), luteotropic hormone (LH) on the day 2-5 of the menstrual cycle, AFC of ovaries. Clinical physical examinations were retrospectively analyzed. Homeostasis model assessment of insulin resistance (HOMA-IR) was evaluation as follows: HOMA-IR $=$ FPG $(\mathrm{mM}) \times$ FINS $(\mathrm{mIU} / \mathrm{L}) / 22.5[18]$.

\section{Retrieval of ovarian granulosa cells (GCs)}

After adequate follicular development was observed by transvaginal ultrasound, human chorionic gonadotropin (hCG) was administered to induce ovulation. Then, 36 hours after using hCG, the oocytes were retrieved by transvaginal ultrasound guided aspiration and the fresh follicular fluid was collected at the same time. Ovarian GCs floating in the follicular fluid immediately were retrieved after arriving at the laboratory on the day. According to previous reports, for the purpose of collecting more pure ovarian GCs from follicular fluid, the magnetic-activated cell sorting(MACS) method was used to retrieve ovarian GCs from fresh follicular fluid[19].

Under the guidance of the manufacturer's instructions, the fresh follicular fluid was centrifuged at 2000rcf for 10 minutes. The supernatant was discarded. Then $1 \mathrm{ml}$ HANKS Balanced Salt Mixture (Solarbio, China) containing $1 \mathrm{mg} / \mathrm{ml}$ Hyaluronidase (Solarbio, China) was added to the sample tube. The mixture was incubated at $37^{\circ} \mathrm{C}$ water for 20 minutes. At the meantime, a $15 \mathrm{ml}$ sample tube which containing $4 \mathrm{ml}$ of Human Peripheral Blood Lymphocyte Separation Solution (TBD) (tbdscience, China) was prepared. Then the mixture was added to the upper liquid. Pay attention to avoiding to mix with the underlying liquid. The mixture was centrifuged at 1700rcf for 10 minutes. Then the ovarian GCs which floating in the middle of the liquid was collected and went through a Pre-Separation Filters (70um) (MACS, Germany) to eliminate cell clumps that may be inserted into the column. Counting the number of GCs after filtering. And the mixture was centrifuged at 6000rcf for 5 minutes, the supernatant was discarded. Then we resuspended the cell pellet was resuspended by 80ul autoMACS Running Buffer (MACS, Germany) and 20ul CD45+ Microbeads human (MACS, Germany) per 10 cells. The mixture was incubated in the dark at $4^{\circ} \mathrm{C}$ water for 15 minutes. $2 \mathrm{ml}$ buffer per $10^{7}$ cells was added into the mixture and the unmarked cells was collected after the mixture went through LS columns (MACS, Germany). The collected GCs suspension was centrifuged again at 6000rcf for 5 minutes, and the cells was transferred to a new microfuge tube by PBS resuspending. The new microfuge tube with GCs suspension was centrifuged at 6000rcf for 3 minutes. Fresh GCs were stored at $-80^{\circ} \mathrm{C}$ after discarding the supernatant.

\section{The exaction of RNA and qRT-PCR}

Take out GCs from $-80^{\circ} \mathrm{C}$ and thaw quickly. Trizol Reagent (Life Technologies, Shanghai, China) was used to isolate total RNA from GCs according to the manufacturer's instructions. The purity and concentration of total RNA were assessed by Nanodrop-2000 (Thermo Fisher Scientific, American) at the absorbance of $260 \mathrm{~nm} / 280 \mathrm{~nm}$. Qualified RNA was reverse transcribed to cDNA by using the Prime Script RT Reagent Kit (TaKaRa, China) according to the protocol.

Quantitative Real Time-PCR (qRT-PCR) was carried out by TB Green ${ }^{\mathrm{TM}}$ Premix Ex Taq ${ }^{\mathrm{TM}}$ II (TaKaRa, China) under the guidance of the instructions and performed on Roche Light Cycle 480 (Hoffman-La Roche). GAPDH was used as the reference gene to normalized the expression of LRH-1. And the $2^{--\Delta C T}$ method was applied to calculate the relative expression of genes.The PCR primers for RT-PCR were all listed in Table 1. 
Table1.Sequences of primers used for qRT-PCR

\section{Statistical analysis}

Kolmogorov-Smirnov test was used to performed normality test of continuous variables. Mean \pm standard deviation (SD) was applicated as the display form of normally distributed variables and assessed the linear association by using Pearson Correlation analysis. The data was analyzed by using the Student's t-test to determine statistical significant. SPSS 26.0 (SPSS, Chicago, IL, USA) and GraphPad Prism 8.0 (GraphPad Software, CA, USA) was used to analyze the data, the P-value $<0,05$ was considered statistically significant.

\section{Result}

\section{Clinical baseline characteristics}

A total of 146 patients participated in this study, including 72 PCOS patients and 74 control patients. The clinical baseline characteristics were summarized in the Table 2. There were no statistically significant differences in age /BMI/HOMA-IR between two groups in order to exclude the influence of age, weight, and glucose metabolism. However, AFC, the level of AMH, LH, FSH, T, DHE-S were statistically significant differences between PCOS group and control group. This was related to the clinical features of PCOS.

Table 2. Clinical baseline characteristics between PCOS group and control group

\section{The comparison of the expression of LRH-1}

The expression of LRH-1 in ovarian granulosa cells was detected in the two groups by qRT-PCR. Compared with the control patients, the expression of LRH-1 was significantly increased in the PCOS patients[(1.03 \pm 0.32$) \mathrm{vs}(1.38 \pm 0.47), \mathrm{t}=5.327, \mathrm{P}<0.0001]$.

Fig.1 The relative expression of LRH-1 in ovarian granulosa cells of PCOS and control patients $(* * * \mathrm{P}<0.0001)$

\section{The correlation between the expression of LRH-1 and the clinical characteristics of patients.}

After analyzing the correlation between the expression level of LRH-1 in ovarian granulosa cells and the clinical data of the recruited patients in the study, it was found that there was a linear correlation between the expression level of LRH-1 with AFC $(r=0.3607, p<0.0001)$ and the level of AMH $(\mathrm{r}=0.2662, \mathrm{p}=0.0012), \mathrm{LH}(\mathrm{r}=0.2518, \mathrm{p}=0.0022)$ and $\mathrm{T}(\mathrm{r}=0.2516, \mathrm{p}=0.0022)$ in all patients. With the increasing of AFC, the expression level of LRH-1 also raised significantly. LRH-1 expression level was positively correlated with the level of AMH, LH, T. At the same time, we also conducted the correlation analysis between the expression level of LRH-1 with other clinical characteristics. However, the results showed that there was no statistical significance between LRH-1 and BMI $(r=0.0791$, $\mathrm{p}=0.6646), \quad$ estradiol$(\mathrm{r}=0.0291, \quad \mathrm{p}=0.7269), \quad \mathrm{FSH}(\mathrm{r}=0.081, \mathrm{p}=0.2901)$, HOMA-IR( $(\mathrm{r}=0.0364, \mathrm{p}=0.3426)$, progesterone $(\mathrm{r}=0.1001, \mathrm{p}=0.2307)$, DHE-S $(\mathrm{r}=0.0756, \mathrm{p}=0.3643)$.

Fig.2 Scatter diagram and linear correlation analysis of LRH-1 expression and AFC, AMH, LH, T in all follicular fluid sample $(\mathrm{n}=146)$

Fig.3 Scatter diagram and linear correlation analysis of LRH-1 expression and BMI, E2, FSH, 
HOMA-IR, Progesterone, DHE-S in all follicular fluid sample $(\mathrm{n}=146)$

\section{Discussion}

In this research, we found that the expression level of LRH-1 in ovarian granulosa cells in PCOS patients had a significant increase compared with the control group. After the correlation analysis between the expression of LRH-1 with the corresponding clinical data, it was found that there was a positively correlation between the expression level of LRH-1 and the AFC, AMH, LH, and T. As we all known, AFC and AMH are often used as clinical observation indicators that can reflect the ovarian reserve.And The LH and T levels were significantly higher in PCOS patients compared with non-PCOS patients[20].

Previous studies have confirmed that LRH-1, as a member of the nuclear receptor family, participated in the development of many cancers. LRH-1 acted as a promising prognostic biomarker and predictor of metastasis non-small cell lung cancer[13]. Research suggested that LRH-1 promoted tumor growth and metastasis of gastric cancer AGS cells[21]. LRH-1 also plays a mediating role in regulating cells stemness. Lai et al. reported GATA6 createed a metabolic symbiosis through up-regulating the expression of LRH-1 to enhances the stemness of human colon cancer cells [22]. In addition, LRH-1 is involved in many metabolic processes, such as the metabolism of glucose, lipids, and bile acids[23-25]. A lot of studies have confirmed that PCOS is associated with an increased prevalence of dysglycemia[26]. Based on the discovery of elevated LRH-1 in PCOS patients, by analyzing the clinical characteristics of recruited patients in this study, we found that PCOS patients have higher levels of AMH, LH, DHES, T and a greater number of antral follicles compared with the control group. It not only suggested that there are disorders in the metabolism and endocrine in PCOS patients, but also implyed that LRH-1 might play an important role in the pathophysiological process of PCOS.

There were also many reports about the research of LRH-1 in female reproductive system. LRH-1 was overexpressed in granulosa cell tumors of the ovary[12], and it was essential for luteinization in the female mouse ovary[27]. Ovary-specific depletion of LRH-1 compromises expansion of the cumulus oophorus[28]. Saxena et al. reported that LRH-1 stimulateed the progesterone biosynthetic pathway during granulosa cell differentiation[10]. By employing a systems biology approach, Bianco et al. revealed that LRH-1 motifs were associated with the early stages of ovulation[29]. Liu et al. discovered that LRH-1 is a critical upstream regulator of CYP19A1 gene expression in ovarian cells, which is essential in the formation of steroid hormines[14]. MiR-1275 could promote granulosa cell apoptosis and repress estradiol synthesis by damaging the LRH-1/CYP19A1 axis in porcine granulosa cells [30].These studies indicated the critical role of LRH-1 playing in the production of progesterone and the process of ovulation. This was consistent with the result in our study that the expression level of LRH-1 in ovarian granulosa cells is positively correlated with AFC and AMH which usually used to reflect ovarian reserve in clinic. A research of $\mathrm{Li}$ et al. found that a $\mathrm{T}>\mathrm{G}$ mutation in the LRH-1 gene was associated with litter size in Hu sheep[31]. LRH-1 deficiency leaded to the compromising placental development and the death of fetal, illustrating it was necessary the presence of LRH-1 for pregnancy[11]. Although there are many research reports on LRH-1, there are no previous studies have linked LRH-1 with the pathogenesis of PCOS.

\section{Conclusion}

For the first time, our study has focus to the relationship between LRH-1 and PCOS, and linked them together, which providing new ideas for the clinical diagnosis and treatment of PCOS. But it also had 
limitations, for example the relatively small number of samples included. In order to better understand the role of LRH-1 in the pathogenesis of PCOS, further basic experimental research is still expected.

\section{Acknowledgments}

The authors thank all the patients who participated in the study.

\section{Reference}

1. F., H., Escobar-Morreale.: Polycystic ovary syndrome: definition, aetiology, diagnosis and treatment. Nat Rev Endocrinol 14(5), 270-284 (2018). doi:10.1038/nrendo.2018.24

2. Kakoly, N.S., Khomami, M.B., Joham, A.E., Cooray, S.D., Misso, M.L., Norman, R.J., Harrison, C.L., Ranasinha, S., Teede, H.J., Moran, L.J.: Ethnicity, obesity and the prevalence of impaired glucose tolerance and type 2 diabetes in PCOS: a systematic review and meta-regression. Human Reproduction Update 24(4), 455-467 (2018). doi:10.1093/humupd/dmy007

3. Zhao Luqian, Z.Z., Lou Huiling, Zhu Guodong,Huang Weimin, Zhang Shaogang, Liu Feng: Polycystic ovary syndrome (PCOS) and the risk of coronary heart disease (CHD): a meta-analysis. Oncotarget Jun 7(7(23)), 33715-33721 (2016).

4. Fayard, E., Auwerx, J., Schoonjans, K.: LRH-1: an orphan nuclear receptor involved in development, metabolism and steroidogenesis. Trends Cell Biol 14(5), 250-260 (2004). doi:10.1016/j.tcb.2004.03.008

5. Galarneau L, D.R., Bélanger L.: Assignment of the fetoprotein transcription factor gene (FTF) to human chromosome band 1q32.11 by in situ hybridization. Cytogenet Cell Genet. 82(3-4), 269-270 (1998).

6. Jin, J., Jin, J., Woodfield, S.E., Patel, R.H., Jin, N.G., Shi, Y., Liu, B., Sun, W., Chen, X., Yu, Y., Vasudevan, S.A.: Targeting LRH1 in hepatoblastoma cell lines causes decreased proliferation. Oncol Rep 41(1), 143-153 (2019). doi:10.3892/or.2018.6793

7. Baquie, M., St-Onge, L., Kerr-Conte, J., Cobo-Vuilleumier, N., Lorenzo, P.I., Jimenez Moreno, C.M., Cederroth, C.R., Nef, S., Borot, S., Bosco, D., Wang, H., Marchetti, P., Pattou, F., Wollheim, C.B., Gauthier, B.R.: The liver receptor homolog-1 (LRH-1) is expressed in human islets and protects \{beta\}-cells against stress-induced apoptosis. Hum Mol Genet 20(14), 2823-2833 (2011). doi:10.1093/hmg/ddr193

8. Botrugno, O.A., Fayard, E., Annicotte, J.-S., Haby, C., Brennan, T., Wendling, O., Tanaka, T., Kodama, T., Thomas, W., Auwerx, J., Schoonjans, K.: Synergy between LRH-1 and $\beta$-Catenin Induces G1 Cyclin-Mediated Cell Proliferation. Molecular Cell 15(4), 499-509 (2004). doi:10.1016/j.molcel.2004.07.009

9. Meinsohn, M.C., Morin, F., Bertolin, K., Duggavathi, R., Schoonjans, K., Murphy, B.D.: The Orphan Nuclear Receptor Liver Homolog Receptor-1 (Nr5a2) Regulates Ovarian Granulosa Cell Proliferation. J Endocr Soc 2(1), 24-41 (2018). doi:10.1210/js.2017-00329

10. Saxena, D., Safi, R., Little-Ihrig, L., Zeleznik, A.J.: Liver receptor homolog-1 stimulates the progesterone biosynthetic pathway during follicle-stimulating hormone-induced granulosa cell differentiation. Endocrinology 145(8), 3821-3829 (2004). doi:10.1210/en.2004-0423

11. Zhang, C., Large, M.J., Duggavathi, R., DeMayo, F.J., Lydon, J.P., Schoonjans, K., Kovanci, E., Murphy, B.D.: Liver receptor homolog-1 is essential for pregnancy. Nat Med 19(8), 1061-1066 (2013). doi:10.1038/nm.3192 
12. Chand, A.L., Pathirage, N., Lazarus, K., Chu, S., Drummond, A.E., Fuller, P.J., Clyne, C.D.: Liver receptor homologue-1 expression in ovarian epithelial and granulosa cell tumours. Steroids 78(7), 700-706 (2013). doi:10.1016/j.steroids.2013.03.001

13. Liu, Y., Xing, Y., Wang, H., Yan, S., Wang, X., Cai, L.: LRH1 as a promising prognostic biomarker and predictor of metastasis in patients with non-small cell lung cancer. Thorac Cancer 9(12), 1725-1732 (2018). doi:10.1111/1759-7714.12887

14. Bayrer, J.R., Mukkamala, S., Sablin, E.P., Webb, P., Fletterick, R.J.: Silencing LRH-1 in colon cancer cell lines impairs proliferation and alters gene expression programs. Proc Natl Acad Sci U S A 112(8), 2467-2472 (2015). doi:10.1073/pnas.1500978112

15. Xu, Z., Hou, X., Lv, H., Sun, B., Cui, Y., Liu, L., Rong, F.: Expression of Liver Receptor Homolog-1 (LRH-1) in Villi and Decidua of Patients with Unexplained Recurrent Spontaneous Abortion. Med Sci Monit 23, 2445-2452 (2017). doi:10.12659/msm.904645

16. Gu, P., Goodwin, B., Chung, A.C., Xu, X., Wheeler, D.A., Price, R.R., Galardi, C., Peng, L., Latour, A.M., Koller, B.H., Gossen, J., Kliewer, S.A., Cooney, A.J.: Orphan nuclear receptor LRH-1 is required to maintain Oct4 expression at the epiblast stage of embryonic development. Mol Cell Biol 25(9), 3492-3505 (2005). doi:10.1128/MCB.25.9.3492-3505.2005

17. Group, T.R.E.A.-S.P.C.W.: Revised 2003 consensus on diagnostic criteria and long-term health risks related to polycystic ovary syndrome. Fertil Steril.(Jan;81(1)), 19-25 (2004). doi:10.1016/j.fertnstert.2003.10.004

18. Kaur, S., Archer, K.J., Devi, M.G., Kriplani, A., Strauss, J.F., 3rd, Singh, R.: Differential gene expression in granulosa cells from polycystic ovary syndrome patients with and without insulin resistance: identification of susceptibility gene sets through network analysis. J Clin Endocrinol Metab 97(10), E2016-2021 (2012). doi:10.1210/jc.2011-3441

19. Ferrero, H., Delgado-Rosas, F., Garcia-Pascual, C.M., Monterde, M., Zimmermann, R.C., Simon, C., Pellicer, A., Gomez, R.: Efficiency and purity provided by the existing methods for the isolation of luteinized granulosa cells: a comparative study. Hum Reprod 27(6), 1781-1789 (2012). doi:10.1093/humrep/des096

20. Wang, L., Fan, H., Zou, Y., Yuan, Q., Hu, X., Chen, X., Zhu, C., Zhang, X., Cui, H.: Aberrant Expression of Long Non-coding RNAs in Exosomes in Follicle Fluid From PCOS Patients. Frontiers in Genetics 11 (2021). doi:10.3389/fgene.2020.608178

21. Liu, L., Li, Y., Pan, B., Zhang, T., Wei, D., Zhu, Y., Guo, Y.: Nr5a2 promotes tumor growth and metastasis of gastric cancer AGS cells by Wnt/beta-catenin signaling. Onco Targets Ther 12, 2891-2902 (2019). doi:10.2147/OTT.S201228

22. Lai, H.T., Chiang, C.T., Tseng, W.K., Chao, T.C., Su, Y.: GATA6 enhances the stemness of human colon cancer cells by creating a metabolic symbiosis through upregulating LRH-1 expression. Mol Oncol (2020). doi:10.1002/1878-0261.12647

23. Lee, J.M., Lee, Y.K., Mamrosh, J.L., Busby, S.A., Griffin, P.R., Pathak, M.C., Ortlund, E.A., Moore, D.D.: A nuclear-receptor-dependent phosphatidylcholine pathway with antidiabetic effects. Nature 474(7352), 506-510 (2011). doi:10.1038/nature10111

24. Choi, S., Dong, B., Lin, C.J., Heo, M.J., Kim, K.H., Sun, Z., Wagner, M., Putluri, N., Suh, J.M., Wang, M.C., Moore, D.D.: Methyl-Sensing Nuclear Receptor Liver Receptor Homolog-1 Regulates Mitochondrial Function in Mouse Hepatocytes. Hepatology 71(3), 1055-1069 (2020). doi:10.1002/hep.30884

25. Miranda, D.A., Krause, W.C., Cazenave-Gassiot, A., Suzawa, M., Escusa, H., Foo, J.C., Shihadih, 
D.S., Stahl, A., Fitch, M., Nyangau, E., Hellerstein, M., Wenk, M.R., Silver, D.L., Ingraham, H.A.: LRH-1 regulates hepatic lipid homeostasis and maintains arachidonoyl phospholipid pools critical for phospholipid diversity. JCI Insight 3(5) (2018). doi:10.1172/jci.insight.96151

26. Li, X., Zhang, T., Li, S., Deng, Y., Wang, L., Tao, T., Wang, S., Gu, Y., Gu, W., Hong, J., Liu, W., Wang, W., Zhang, Y.: Correlation between glucose metabolism and serum steroid hormones in patients with polycystic ovary syndrome. Clinical Endocrinology 92(4), 350-357 (2020). doi:10.1111/cen.14154

27. Bertolin, K., Gossen, J., Schoonjans, K., Murphy, B.D.: The orphan nuclear receptor Nr5a2 is essential for luteinization in the female mouse ovary. Endocrinology 155(5), 1931-1943 (2014). doi:10.1210/en.2013-1765

28. Bertolin, K., Meinsohn, M.C., Suzuki, J., Gossen, J., Schoonjans, K., Duggavathi, R., Murphy, B.D.: Ovary-specific depletion of the nuclear receptor $\mathrm{Nr} 5 \mathrm{a} 2$ compromises expansion of the cumulus oophorus but not fertilization by intracytoplasmic sperm injection. Biol Reprod 96(6), 1231-1243 (2017). doi:10.1093/biolre/iox045

29. Bianco, S., Bellefleur, A.M., Beaulieu, E., Beauparlant, C.J., Bertolin, K., Droit, A., Schoonjans, K., Murphy, B.D., Gevry, N.: The Ovulatory Signal Precipitates LRH-1 Transcriptional Switching Mediated by Differential Chromatin Accessibility. Cell Rep 28(9), 2443-2454 e2444 (2019). doi:10.1016/j.celrep.2019.07.088

30. Liu, J., Li, X., Yao, Y., Li, Q., Pan, Z., Li, Q.: miR-1275 controls granulosa cell apoptosis and estradiol synthesis by impairing LRH-1/CYP19A1 axis. Biochim Biophys Acta Gene Regul Mech 1861(3), 246-257 (2018). doi:10.1016/j.bbagrm.2018.01.009

31. Li, Y., Zhang, J., Qian, Y., Meng, C., Wang, H., Zhong, J., Cao, S.: A T > G Mutation in the NR5A2 Gene Is Associated With Litter Size in Hu Sheep Through Upregulation of Promoter Activity by Transcription Factor MTF-1. Front Genet 10, 1011 (2019). doi:10.3389/fgene.2019.01011 
Figures

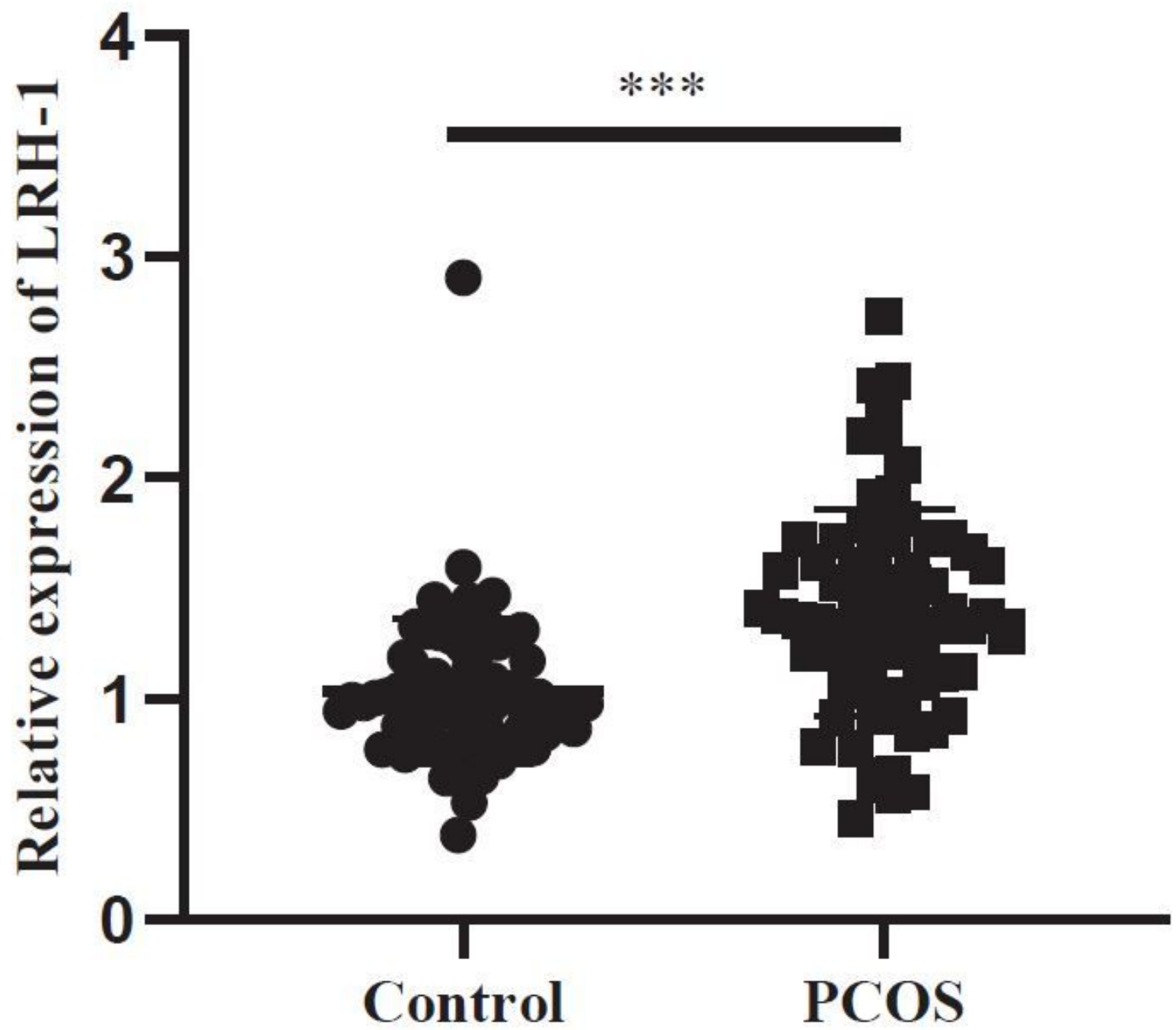

Figure 1

The relative expression of $\mathrm{LRH}-1$ in ovarian granulosa cells of PCOS and control patients $\left({ }^{\star \star \star} \mathrm{P}<0.0001\right)$ 
a
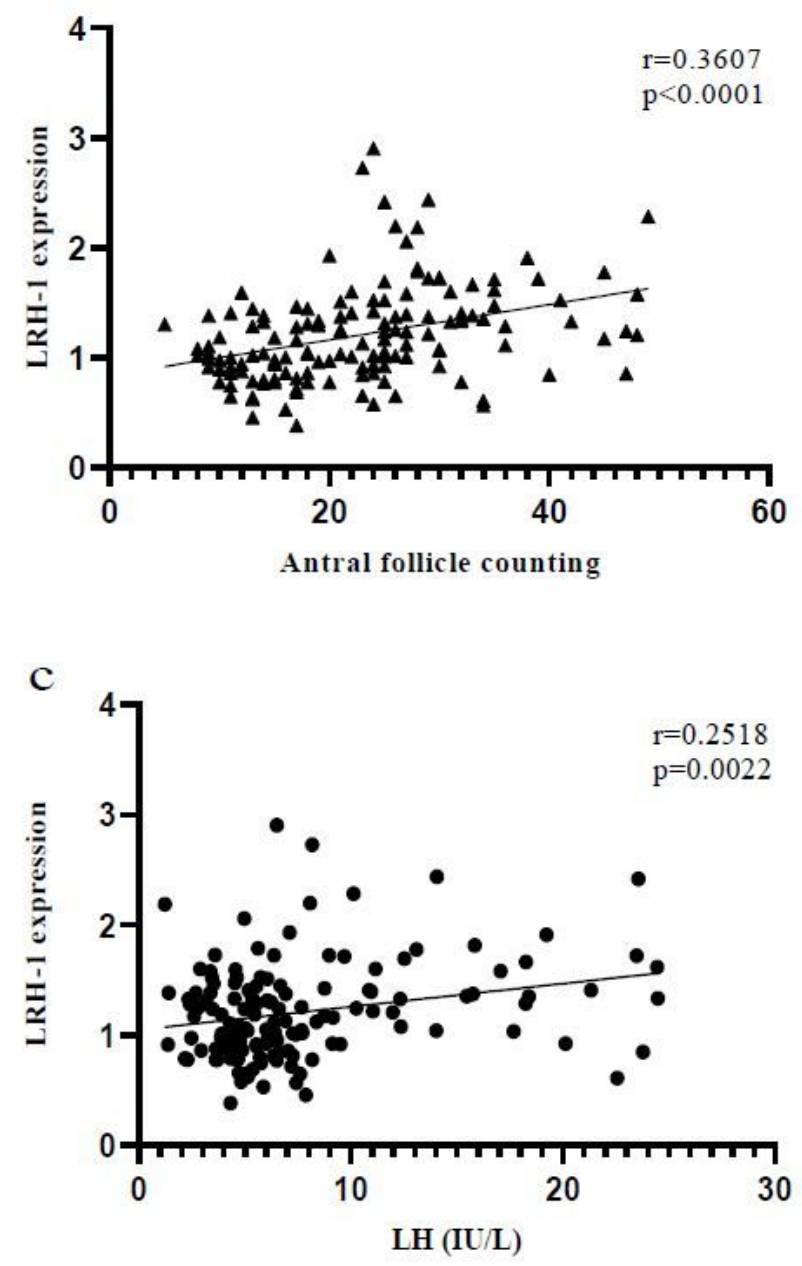

b
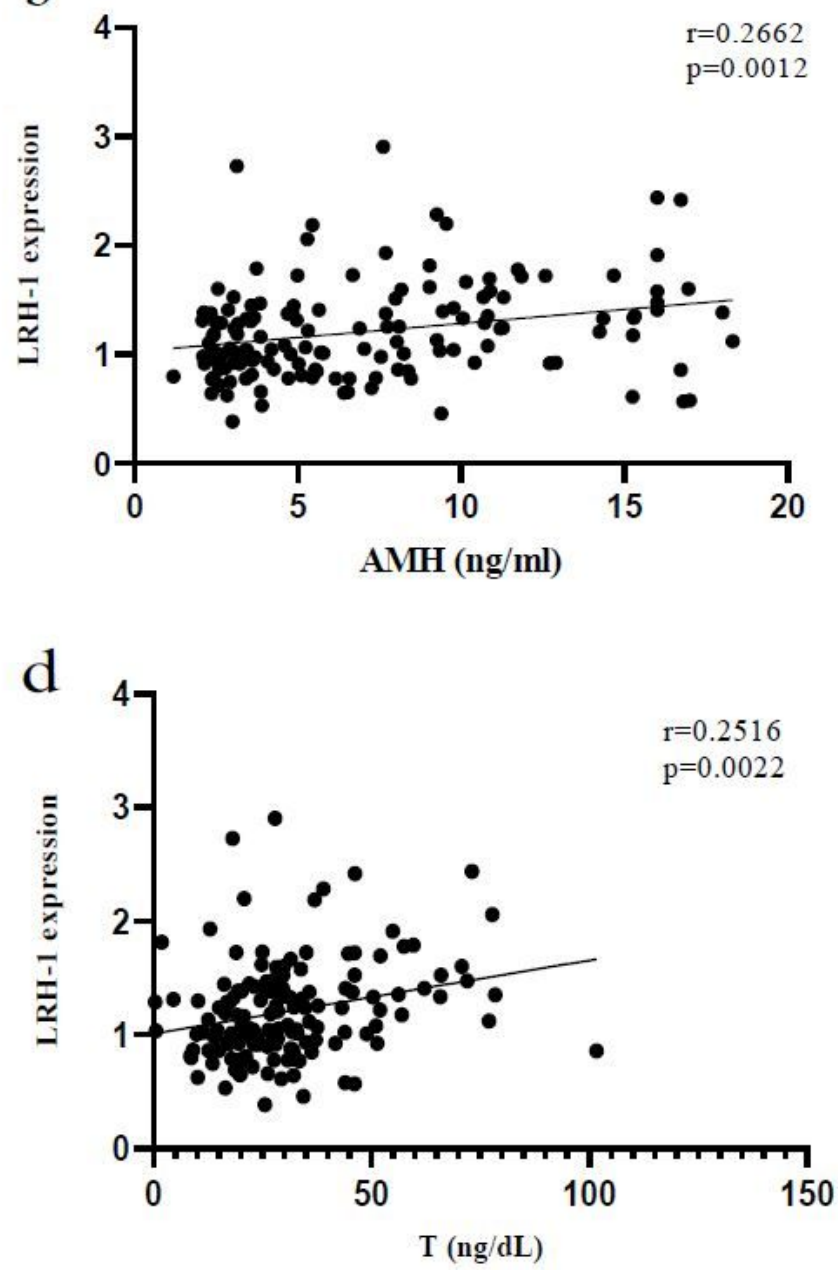

Figure 2

Scatter diagram and linear correlation analysis of LRH-1 expression and AFC, AMH, LH, T in all follicular fluid sample ( $n=146$ ) 

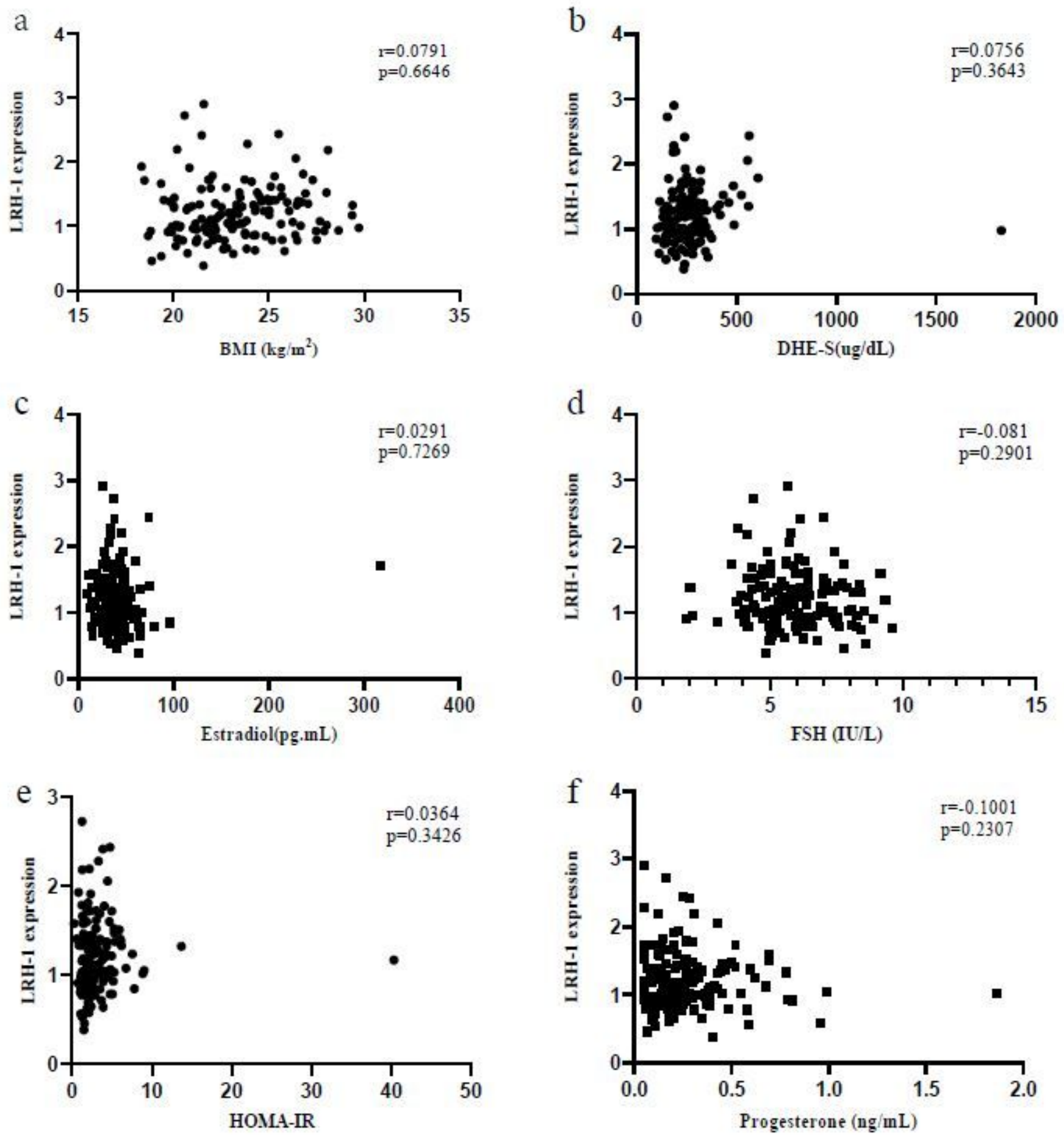

Figure 3

Scatter diagram and linear correlation analysis of LRH-1 expression and BMI, E2, FSH, HOMA-IR, Progesterone, DHE-S in all follicular fluid sample $(n=146)$

\section{Supplementary Files}


This is a list of supplementary files associated with this preprint. Click to download.

- Table.1.pdf

- Table.2.pdf 